\title{
Rhabdomyosarcoma in adolescents: A CCHE experience
}

\author{
Emad A.H Moussa ${ }^{1}$, Enas El Nadi ${ }^{2}$, Hosam El Zomor ${ }^{3}$, Hanafy Ahmed Hafez ${ }^{3}$, Alaa Younes ${ }^{4}$, Hala \\ Taha $^{5}$, Naglaa EL-Kenanii ${ }^{5}$, Mohamed S. Zaghloul ${ }^{6}$, Madeha El-Wekeel ${ }^{7}$, Rania M. Labib ${ }^{8}$, Marwa \\ Tantawy ${ }^{8}$
}

1. Faculty of medicine, Menufeya University, Egypt. 2. Faculty of medicine, Beni-Suef University, Egypt. 3. Pediatric Oncology, $\mathrm{NCl}$-Cairo University, Egypt. 4. Surgical oncology, $\mathrm{NCl}$-Cairo University, Egypt. 5. Pathology, NCl-Cairo University, Egypt. 6. Radiation Oncology, $\mathrm{NCl}$-Cairo University, Egypt. 7. Radiology NCl-Cairo University, Egypt. 8. CRA at Research Dept CCHE, Egypt.

Correspondence: Emad A.H Moussa. Address: Faculty of medicine, Menufeya University, Egypt.

Email: moussaemad1960@yahoo.co.uk

Received: November 5, 2014 Accepted: February 27, 2015 Online Published: April 13, 2015

DOI : $10.5430 /$ jst.v5n2p1

URL: http://dx.doi.org/10.5430/jst.v5n2p1

\section{Abstract}

Objective and aim: A lower outcome of rhabdomyosarcoma in elder children and young adolescents is increasingly recognized. Our aim was to focus on the outcome of elder children and adolescents with rhabdomyosarcoma treated at our Center (CCHE).

Patients and methods: Patients between the age 10 and 18 years diagnosed as rhabdomyosarcoma and treated according to IRS -IV, or IRS V protocols determined according to site, stage, and age at diagnosis.

Results: Thirty-two children were recruited at Children's Cancer Hospital-Egypt. They were 12 females (37.5\%) and 20 males (62.5\%), with a mean age of 12.8y. The mean follow up period was 29.4 months (July 2007 till December 2012). Seventeen patient, were allocated to IRS-IV, and 15 patient to IRS-V. The overall survival and failure free survival were $59.1 \%$ and $40.4 \%$ respectively. Forty six percent of patients had alveolar pathology, $68.8 \%$ presented with tumors at unfavorable sites; $37.5 \%$ had metastases at presentation; and $21.9 \%$ had intracranial extension at presentation. Risk status, clinical grouping and tumor size affected FFS significantly. Local control was done by radiation therapy in $62.5 \%$, surgery alone in $9.4 \%$, and both radiation and surgery in $25 \%$.

Conclusion: The outcome of adolescent group showed inferior results compared to younger children. More cooperation and knowledge about RMS, in this age category, can help develop more intensive unified management approach for them.

\section{Key words}

Rhabdomyosarcoma, Adolescents, Sarcoma, Survival

\section{Background}

Rhabdomyosarcoma occurs predominantly in children below 7 years, with another peak in adolescence, then the incidence declines in older patients ${ }^{[1,2]}$. 
Survival has improved much in children with rhabdomyosarcoma ${ }^{[3,4]}$, especially for non-metastatic disease. A lower outcome for older children and young adults with RMS is expected. Age is an important factor to consider as it greatly influences treatment decisions and possibilities. The treatment strategy use multi-agent chemotherapy, with or without local therapy. Although primary or secondary surgical resection, is important for local control, complete resection is not always feasible at certain cases. Radiotherapy (RT) has assumed a major role in the management of many patients ${ }^{[5]}$.

Our aim was to focus on the outcome of our elder children and adolescents with rhabdomyosarcoma treated according to pediatric guidelines.

\section{Patients and methods}

\subsection{Eligibility and consent}

Patients aged from 10 to 18 years diagnosed as rhabdomyosarcoma were eligible provided they received no prior treatment, and started chemotherapy within 4 weeks of the date of initial biopsy or surgery. Informed consent was obtained from parent, according to our institutional review board (IRB) guidelines.

\subsection{Staging}

The disease was evaluated by standard clinical and radiologic techniques. Computed tomography (CT) or MRI were done to assess disease locally and regionally as well as metastatic workup including CT chest, bone scan, bilateral bone marrow aspirate and biopsy and PET assessment for some cases. CSF examination was done for all parameningeal tumors.

Biopsy was done for all presented cases for primary lesion or any suspicious lymph node involvement. Pathological assessment was done using $\mathrm{H} \& \mathrm{E}$ and immunohistochemical studies.

Staging and clinical grouping were done to all cases. Staging was done according to TNM staging and based on IRS-V guidelines (see Table 1).

Table 1. Pretreatment TNM staging system for RMS

\begin{tabular}{llllll}
\hline $\mathbf{M}$ & $\mathbf{N}$ & Size & $\mathbf{T}$ & Sites & Stage \\
\hline $\mathrm{M}_{0}$ & $\mathrm{~N}_{0}$ or $\mathrm{N}_{1}$ or $\mathrm{N}_{\mathrm{x}}$ & a or $\mathrm{b}$ & $\mathrm{T}_{1}$ or $\mathrm{T}_{2}$ & $\begin{array}{l}\text { Orbit, head and neck (excluding parameningeal), genitourinary } \\
\text { (nonbladder/nonprostate), biliary tract }\end{array}$ & 1 \\
$\mathrm{M}_{0}$ & $\mathrm{~N}_{0}$ or $\mathrm{N}_{\mathrm{x}}$ & $\mathrm{a}$ & $\mathrm{T}_{1}$ or $\mathrm{T}_{2}$ & $\begin{array}{l}\text { Bladder/prostate, extremity, cranial, parameningeal, other (includes } \\
\text { trunk, retroperitoneum, etc) }\end{array}$ \\
$\mathrm{M}_{0} \mathrm{M}_{0}$ & $\mathrm{~N}_{1} \mathrm{~N}_{0}$ or $\mathrm{N}_{1}$ or $\mathrm{N}_{\mathrm{x}}$ & ab & $\mathrm{T}_{1}$ or $\mathrm{T}_{2}$ & $\begin{array}{l}\text { Bladder/prostate, extremity, cranial, parameningeal, other (includes } \\
\text { trunk, retroperitoneum, etc) }\end{array}$ \\
$\mathrm{M}_{1}$ & $\mathrm{~N}_{0}$ or $\mathrm{N}_{1}$ & a or b & $\mathrm{T}_{1}$ or $\mathrm{T}_{2}$ & Any & \\
\hline
\end{tabular}

Notes. Size: a, $\# 5 \mathrm{~cm}$ in diameter; $\mathrm{b}, .5 \mathrm{~cm}$ in diameter.

Abbreviations: TNM, TNM Classification of Malignant Tumors; RMS, rhabdomyosarcoma; T, tumor; $\mathrm{T}_{1}$, confined to anatomic site of origin; $\mathrm{T}_{2}$, extension and or/fixative to surrounding tissue; $\mathrm{N}$, nodes; $\mathrm{N}_{0}$, regional nodes not clinically involved; $\mathrm{N}_{\mathrm{l}}$, regional nodes clinically involved; $\mathrm{N}_{\mathrm{x}}$, regional node status unknown; $\mathrm{M}$, metastasis; $\mathrm{M}_{0}$, no distant metastasis; $\mathrm{M}_{1}$, metastasis present (includes positive cytology in pleural, peritoneal, or cerebrospinal fluid).

All diagnoses by our pathologists were classified according to the international classification ${ }^{[6]}$.

\subsection{Treatment schedule}

Patients were treated with combined modality treatment including surgery, multi-agent chemotherapy and/or radiotherapy, adapted according to Risk stratification. 
Therapy was assigned based on the IRSG rhabdomyosarcoma risk group classification (see Table 2). All our Patients were categorized according to the stage, clinical group and histological subtype into:

1) Low risk group: Included patients with embryonal RMS or botryoid who had:

- Non-metastatic tumors arising in favorable sites (stage 1), clinical group I, II, or III

- Non-metastatic tumors in unfavorable sites (stage 2 or 3 ) that are grossly resected with or without microscopic residual (clinical group I or II)

2) Intermediate risk group: Included patients with:

- Embryonal RMS or botryoid who had stage 2 or 3 and clinical group III

- Alveolar RMS who had stage 1, 2, or 3 and clinical group I, II, or III

- Non metastatic Parameningeal primary site regardless the histology who had clinical group I, II, or III

3) High risk group: Included all metastatic patients with stage 4

Table 2. COG clinical group classification of RMS

\begin{tabular}{ll}
\hline Definition & Group \\
\hline Localized disease, completely resected & Group I \\
Total gross resection, with evidence of regional spread & Group II \\
Grossly resected tumor with microscopic residual disease & A \\
Involved regional nodes completely resected with no microscopic residual disease & B \\
Involved regional nodes grossly resected with evidence of microscopic residual disease & C \\
Biopsy only or incomplete resection with gross residual disease & Group III \\
Distant metastatic disease (excludes regional nodes and adjacent organ infiltration) & Group IV \\
\hline
\end{tabular}

Note. COG, Children's Oncology Group; RMS, rhabdomyosarcoma

\subsection{Chemotherapy}

Figure 1 shows Summary of treatment protocol (Low Risk):

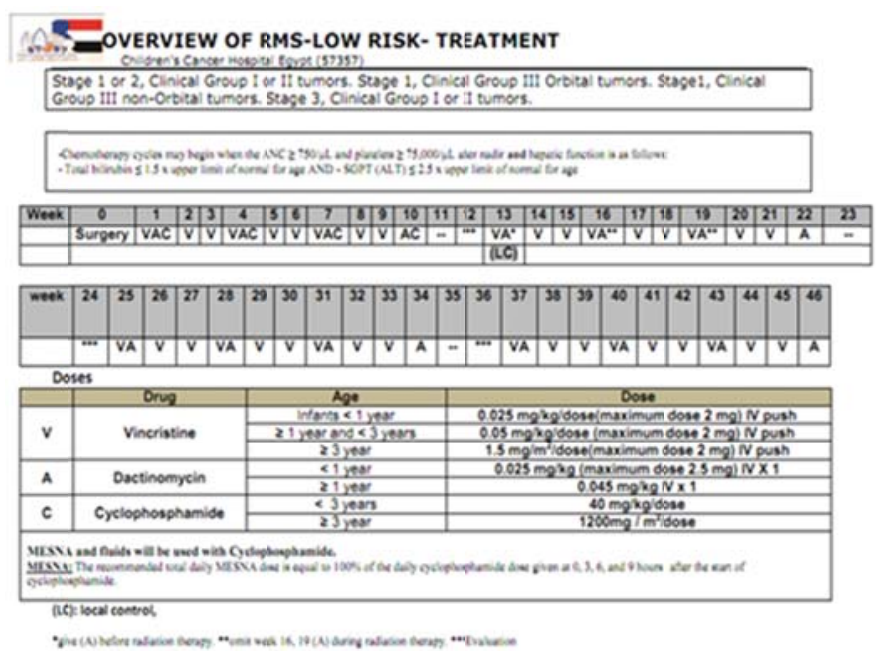

Figure 1. Treatment protocol (low risk) 
Figure 2 shows treatment protocol (Intermediate Risk):

Figure 2. Treatment protocol (Intermediate risk)

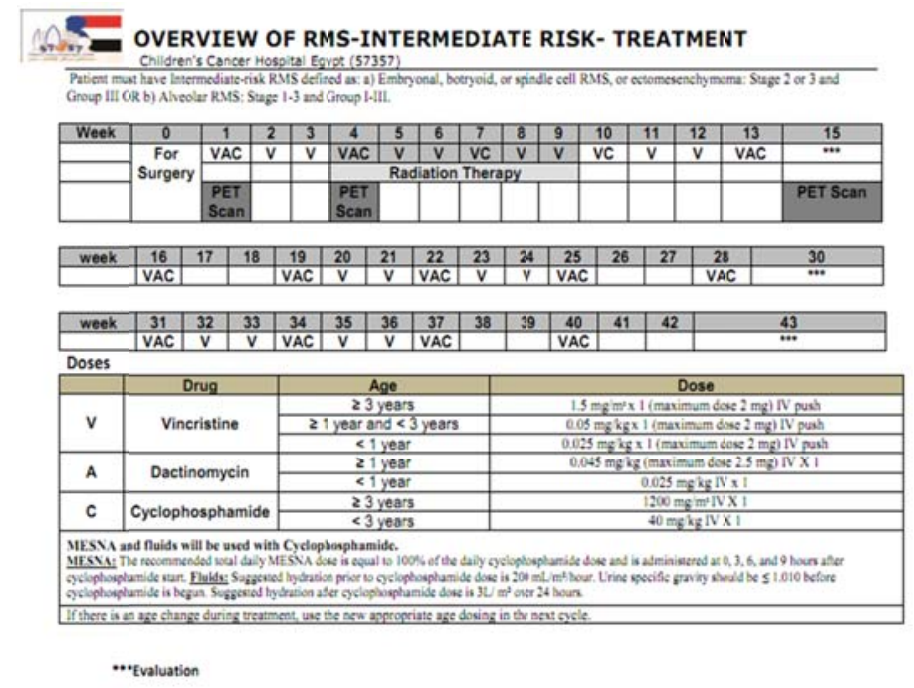

Figure 3 shows treatment protocol (High Risk):

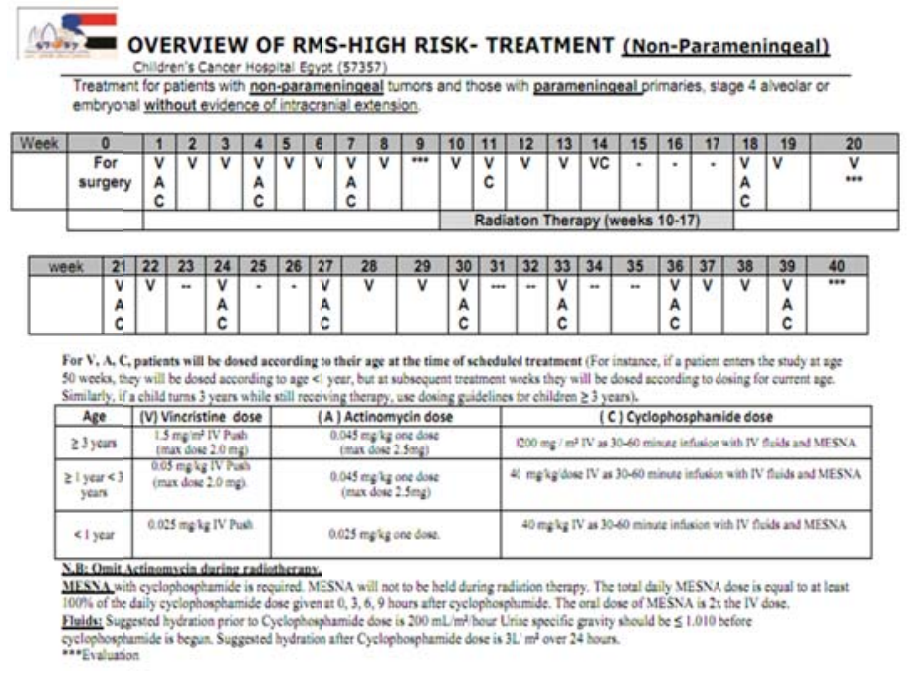

Figure 3. Treatment protocol (High Risk)

\subsection{Local therapy}

A delayed surgery if possible was performed for patients with clinical group III disease on week 12. No radiotherapy was given in non-metastatic embryonal tumors with clinical group I. All other patients received radiotherapy following week 12 except in cases of parameningeal sites with criteria of meningeal extension, where radiotherapy was given on day 0 .

The gross tumor volume (GTV) was defined as the pre-treatment visible and for palpable disease detected by physical examination, operative findings, CT or MRI including any involved lymph nodes. For all clinical groups, the clinical target volume (CTV) was defined by adding one $\mathrm{cm}$ safety margin to GTV. Those who have clinical group III disease who do not undergo a second look operation may have a second CTV defined for a core down boost. The planning tumor volume (PTV) is created by adding safety margins that deals with the setup position uncertainties. Clinical group II (stage I and II) without nodal involvement received $36 \mathrm{~Gy}$, while those with nodal involvement $\left(\mathrm{N}_{1}\right)$ received $41.4 \mathrm{~Gy}$. Clinical group III received 45 Gy if in the orbit and 50.4 Gy in non-orbit sites. Patients received radiotherapy to metastatic sites which can be localized and imaged (i.e. excluding the bone marrow). 


\subsection{Statistical analysis}

Patients' data were tabulated and processed using (SPSS) statistical package ${ }^{[7]}$ for Windows ${ }^{[8]}$. Qualitative data were expressed as frequency and percentage, while quantitative data were expressed as mean SD and median. The chi-square test and Fisher Exact test were used for comparative analysis. Statistically significant level was considered at $p \leq .05$.

Survival was calculated using the Kaplan-Meier method. Patients without adverse events were censored on the date of the last reported contact. The differences between curves were tested for statistical significance using the log rank test ${ }^{[9]}$.

Failure-free survival (FFS) was defined as the time from the start of treatment to disease progression, recurrence, or death as a first event.

Overall survival (OS) was defined as the time from start of treatment to death whatever the cause is.

\section{Results}

At a follow up period ranging from $6.7 \mathrm{~m}$ to $61.3 \mathrm{~m}$ with a mean of 29.4 months from July 2007 till end of December 2012, 32 children and adolescents between 10 and 18 years were newly diagnosed with rhabdomyosarcoma at "The Children's Cancer Hospital-Egypt". They were 12 females (37.5\%) and 20 males (62.5\%), with a mean age of 12.8 year (range 10 -17.7 year) (see Table 3 ).

Table 3. Showing patients' characteristics, and survival

\begin{tabular}{|c|c|c|c|c|}
\hline & OS & $p$-value & FFS & $p$-value \\
\hline \multicolumn{5}{|l|}{ Sex } \\
\hline Male $(\mathrm{n}=20)$ & $75 \%$ & & $36.6 \%$ & \\
\hline Female $(\mathrm{n}=12)$ & $50.3 \%$ & .255 & $47.6 \%$ & 6 \\
\hline \multicolumn{5}{|l|}{ Risk-Status } \\
\hline $\operatorname{High}(\mathrm{n}=12)$ & $19.4 \%$ & & $12.5 \%$ & \\
\hline Intermediate $(\mathrm{n}=17)$ & $69.1 \%$ & & $56.6 \%$ & \\
\hline Low $(n=3)$ & $100 \%$ & .07 & $66.7 \%$ & .03 \\
\hline \multicolumn{5}{|l|}{ Tumor size } \\
\hline$<5 \mathrm{~cm}(\mathrm{n}=10)$ & $100 \%$ & & $77.1 \%$ & \\
\hline$>5 \mathrm{~cm}(\mathrm{n}=18)$ & $35.6 \%$ & & $18.7 \%$ & \\
\hline Unknown $(\mathrm{n}=4)$ & $50 \%$ & .005 & $37.5 \%$ & .008 \\
\hline \multicolumn{5}{|l|}{ Clinical Group } \\
\hline $\mathrm{I}(\mathrm{n}=2)$ & $100 \%$ & & $50 \%$ & \\
\hline III (n=18) & $71 \%$ & & $59.2 \%$ & \\
\hline IV $(n=12)$ & $19.4 \%$ & .08 & $12.5 \%$ & .035 \\
\hline \multicolumn{5}{|l|}{ L.Node involvement } \\
\hline Yes $(n=12)$ & $46.5 \%$ & & $38.9 \%$ & \\
\hline No $(n=20)$ & $90 \%$ & .05 & $39 \%$ & .6 \\
\hline \multicolumn{5}{|l|}{ Pathology } \\
\hline Embryonal ( $\mathrm{n}=17$ ) & $60.4 \%$ & & $33.3 \%$ & \\
\hline Alveolar (n=15) & $60 \%$ & .6 & $46.6 \%$ & .13 \\
\hline \multicolumn{5}{|l|}{ Local control } \\
\hline Radiotherapy only (n=20) & $61.9 \%$ & & $45.3 \%$ & \\
\hline Surgery only $(\mathrm{n}=3)$ & $66.7 \%$ & & $33.3 \%$ & \\
\hline Both $(n=8)$ & $46.9 \%$ & .9 & $37.5 \%$ & .9 \\
\hline
\end{tabular}




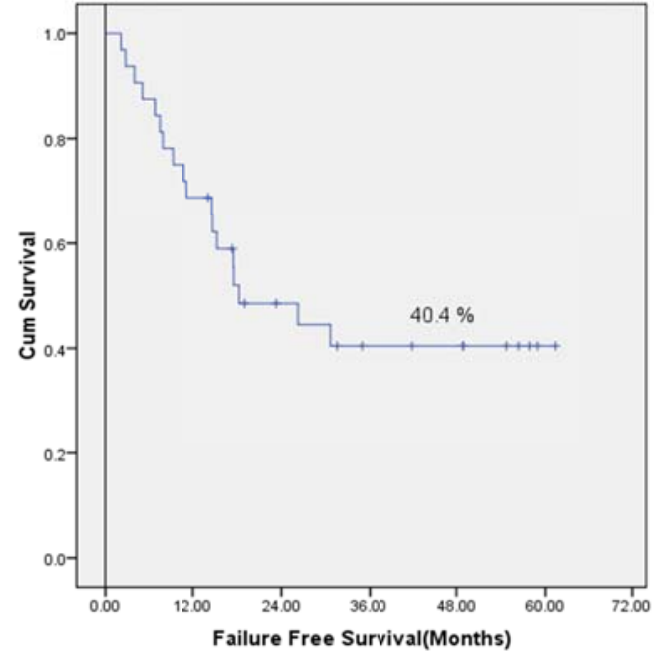

Figure 4. Failure free survival (40.4\%)

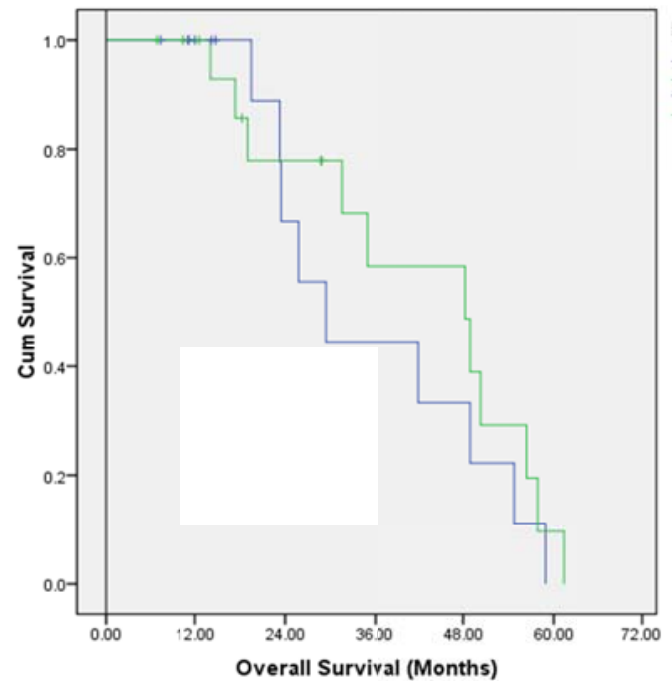

Figure 6. Overall survival for different pathological subtypes

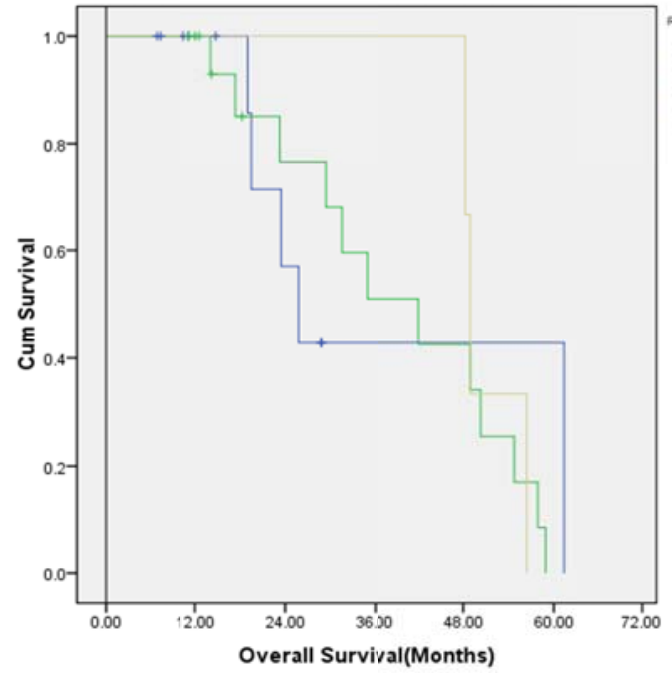

Figure 8. Overall survival of different Risk groups $\rightarrow$ Survival Function
+ Censored
and

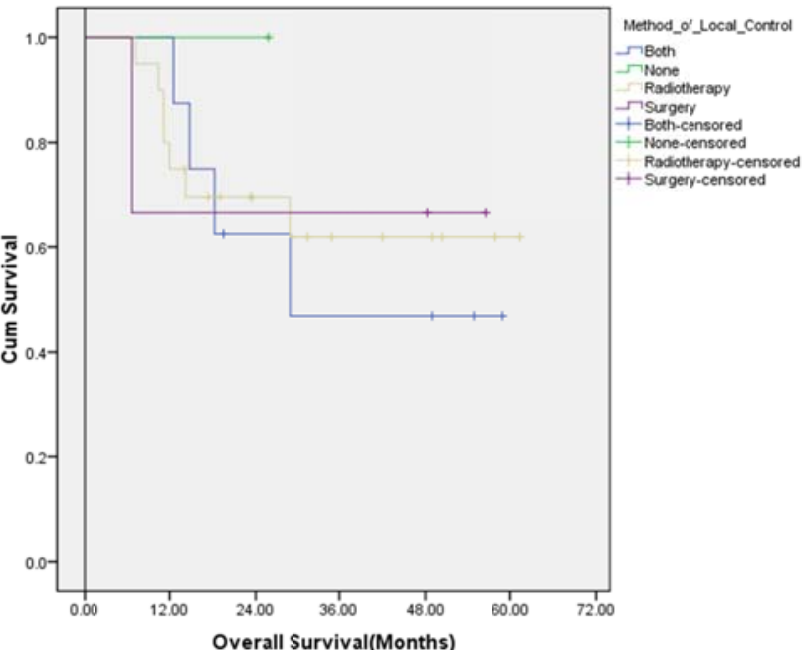

Figure 5. Overall survival for patients categorized by type of local control

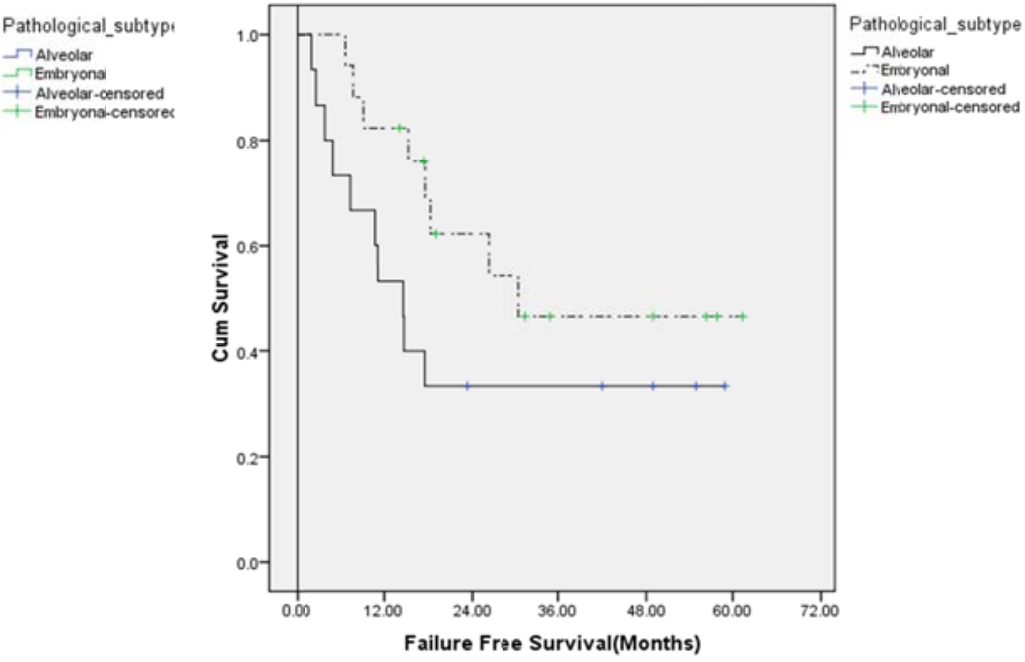

Figure 7. Failure free survival of pathological subtypes
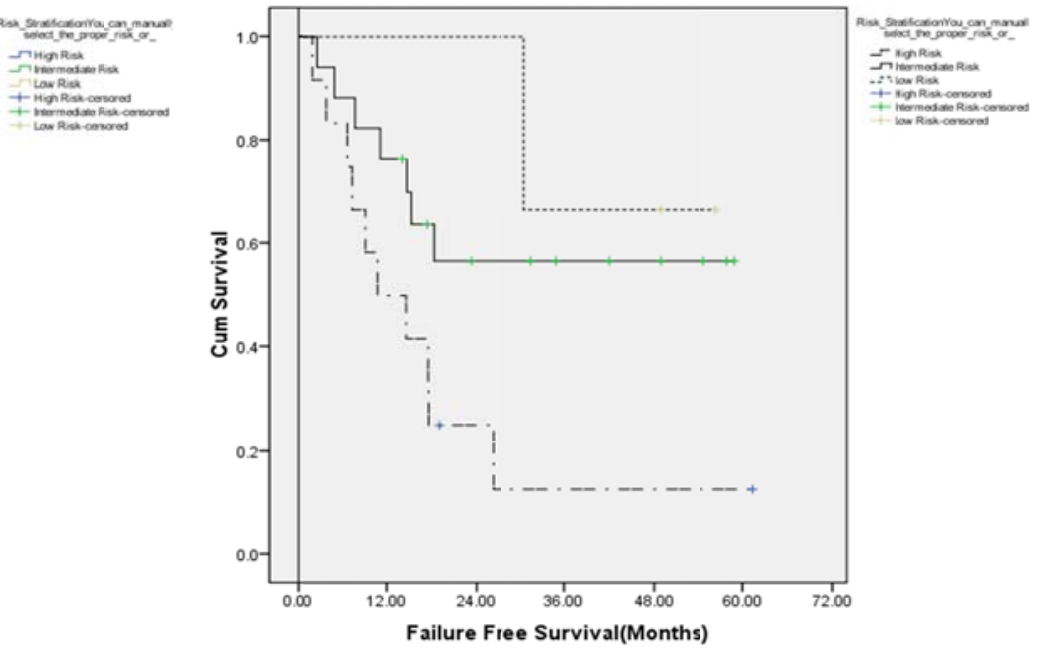

Figure 9. Failure free survival of different Risk groups 
Seventeen patient, were allocated to IRS -IV, and the rest (15 patients) to IRS V treatment schedules by site and stage. We distributed our patients according to tumor site, clinical group, stage, size, median age, and treatment group according to IRS-V guidelines into low, intermediate and high risk groups. Of the 32 children recruited, 15 (46.8\%) patients had alveolar, and 17 patients had embryonal pathology. Seven patients $(21.9 \%)$ had intracranial extension at presentation, while 22 patients $(68.8 \%)$ presented with tumors at unfavorable sites, and 12 patients $(37.5 \%)$ had metastases at presentation. By risk stratification 12 patients (37.5\%) were high risk, 17 (53.1\%) were intermediate risk and only 3 patients $(9.4 \%)$ had low risk disease. Local control was done by radiation therapy in 20 patients $(62.5 \%)$, surgery alone was performed for 3 patients $(9.4 \%)$, and both radiation and surgery in 8 patients $(25 \%)$. One patient received no local control due to early disease progression.

Our patients overall Survival was $59.1 \%$ while failure free survival was $40.4 \%$. Presence of metastases at presentation, showed inferior results significantly $(p=.01)$. Risk stratification, was correlated significantly with failure free survival $(p=.03)$, while tumor size and clinical grouping were statistically significant in overall and failure free survival outcome.

In response to first line chemotherapy, twelve patients (37.5\%) attained C.R, 7 patients (21.9\%) showed P.R, while disease progression was seen in 13 patients (40.6\%). At a mean follow up period of 29.4 months (range $=6.7-61.3$ months) 17 patients $(53.1 \%)$ showed relapse or disease progression post first line chemotherapy, while 15 patients (46.9\%) continued in complete remission (see Figures 4-9).

\section{Discussion}

Adolescent RMS is rare, and it has an inferior outcome if compared to younger patient population.

Improved survival rates occurred over the past years, resulting in a 5-year survival rate of approximately $70 \%-80 \%$ for children with RMS ${ }^{[10-13]}$, while survival rates in adults are not exceeding $56 \%$ (range $\left.21 \%-56 \%\right)^{[14-17]}$.

Our study survival data resulted in a few observations; First, a high percent of patients presented with unfavorable features, as (37.5\%) of the study population presented with metastatic RMS, $46.8 \%$ of patients, had alveolar pathology, $68.8 \%$, had tumors arising in unfavorable sites, and $21.9 \%$ had intracranial extension at presentation which is unexpectedly high. In spite the small number of our studied population which doesn't permit a reliable statistical conclusions, yet this might give an explanation of the inferior outcome for this group of patients.

Second, adolescent patients with RMS showed a lower overall Survival of $59.1 \%$ while failure free survival was $40.4 \%$. This outcome was comparable to results from adult population with RMS, the 5-year rate of OS was $45 \%$ for patients with non-metastatic disease ${ }^{[7]}$. Failure rates for patients with non-metastatic disease at 5 years were $34 \%$ for local failure and $42 \%$ for distant failure ${ }^{[7]}$. Our results also coincide with another study reporting that infants and adolescents were more prone to have unfavorable features, including alveolar pathology, undifferentiated tumors and advanced group or Stage. They also showed a significantly poor FFS than did children aged $1-9$ years $(51 \% \text { vs. } 72 \%, P<.001)^{[18]}$. At a median follow-up of 4.3 years, in a COG study D9803, the 4-year FFS was $73 \%$ with VAC and $68 \%$ with VAC/VTC $(P=.3)^{[19]}$.

Another study, showed a 4 -year EFS being $83 \%$ for alveolar/undifferentiated sarcoma (UDS) stage 1 group I; $74 \%$ for embryonal stage $2 / 3$ group III; $68 \%$ for parameningeal disease with intracranial extension; $60 \%$ for alveolar/UDS stage $2 / 3$ and group II/III patients and $59 \%$ for patients with embryonal group IV disease ${ }^{[8]}$.

Third, most of our patients had either Intermediate (53.1\%) or high (37.5\%) risk categories while only $(9.4 \%)$ had low risk disease. Currently, low-risk patients are defined as patients with an excellent outcome of $85 \%$ to $95 \%$. These are patients with nonmetastatic disease of embryonal histology at favorable sites and at unfavorable sites if totally resected. They have an excellent outcome (2-year event-free survival [EFS] 88\%, OS 98\%) with short duration therapy with low cumulative 
alkylator dose ${ }^{[20]}$. Further classification is done by COG to stratify those patients, of which a patient subset might be futurely transferred to intermediate risk group ${ }^{[21,22]}$.

High-risk patients include all patients with metastatic disease; however, this is also a diverse group with various subsets which differ in prognosis which ranges from $5 \%$ to $50 \% 3$-year EFS ${ }^{[23]}$.

Fourth, almost half of adolescent age group (46.9\%) had pathological subtype "Alveolar", while ERMS is found in approximately $70 \%$ of all childhood RMS. Although the embryonal and alveolar histologic variants are more commonly seen in pediatric patients, the pleomorphic variant is seen more often in adults ${ }^{[2]}$. Few studies focused on the age-related biological and molecular differences of RMS ${ }^{[25]}$. RMS is possibly a different biological and clinical entity in patients aged 10 years or greater. As the subtype and translocation status have prognostic significance, the different pattern of histology across age, for instance the pleomorphic subtype higher presentation, may explain the difference in outcome.

In our study, metastatic tumor in adolescent age group ( $>10$ years), had less 5 -year overall survival or failure free survival than their non metastatic counterparts, and this difference was highly significant (For OS $P=.034$; for FFS $P=.01$ ). This raises the question that patients over 10 years of age with localized disease may be treated less effectively than their counterparts who have metastatic disease at diagnosis.

In one study after adjusting other prognostic factors, age at diagnosis has been shown to be an independent prognostic factor with infants under 1 and children 10 and older having a worse outcome ${ }^{[25]}$.

Several studies mentioned age as a prognostic factor with some emphasizing a strong interrelationship between age, site, and histology ${ }^{[8]}$. In other studies, age and sex had no impact on OS, despite a trend for an increased relative risk in children aged $>10$ years ${ }^{[9]}$, but in this study, a relative under-representation of older patients ( $81 \%$ aged $<10$ years), reduced the power of the analysis ${ }^{[9]}$.

RMS is a malignant tumor, arising from skeletal muscle. Since gross total excision is not always possible, and various studies have advocated aggressive surgical intervention in a belief that reduction of tumor bulk affects the outcome, there are reports indicating very good response either to chemo and radiotherapy, or delayed surgery after chemotherapy ${ }^{[26]}$.

In our study only $9.4 \%$ of adolescent age group did gross total excision while $25 \%$ did partial excision followed by radiation therapy. This, relatively lower portion of patients suffering RMS undergoing surgery, might share in the lower survival as adequate local control is better with proper surgical excision.

None of our patients underwent a second look surgery. Induction chemotherapy, followed by concurrent chemoradiation, is the current standard of care for patients with unresected disease, those with residual disease after surgery for patients with lymph node involvement, and for patients with alveolar histology ${ }^{[27,28]}$.

The major cause of treatment-failure in rhabdomyosarcoma remains inadequate local control. Assessment for initial surgical resection followed by chemotherapy and local RT, with or without further surgery, achieves local control in more than $80 \%$ of patients. Such good results are not achieved at all sites (e.g., trunk and limbs) or all patients (e.g., those with large $[>10 \mathrm{~cm}]$ primary tumors), and failure rates of $11 \%$ to $18 \%$ are still reported even at favorable sites ${ }^{[29]}$. Our data showed $56.3 \%$ to have large tumors $(>5 \mathrm{~cm})$ and that tumor size had a significant impact on OS $(p=.005)$ and FFS $(p=.008)$.

In one study, although outcomes were inferior in adolescents, it was unclear whether the differences in toxicity observed in this study had an impact on outcome. The authors concluded that future studies examining the age-related and sex-related differences in pharmacokinetics of chemotherapy are necessary ${ }^{[30]}$. 
The higher prevalence of unfavorable tumor characteristics noted among adolescents as well as the lack of unified protocols for this category of patients seem to explain their worse outcome compared to children. However, the limited number of our study, enrolling adolescent patients is calling for more cooperation between various oncological centers for conducting more reliable statistically randomized studies addressing this category of patients.

In our conclusion, the outcome of adolescent rhabdomyosarcoma showed inferior results to children when using childhood protocols. An increase in knowledge about RMS, especially biological characteristics in older children and adolescence can help to develop worldwide standardized treatment for this group of patients. Increased use of multidisciplinary therapy may improve older patient clinical outcome.

Despite its limitations, this study highlights the need to improve the management of patients with RMS over 10 years of age. We should ensure that these patients benefit as much from clinical management and research progress as younger patients with RMS.

\section{References}

[1] Punyko JA, Mertens AC, Baker KS, et al. Long-term survival probabilities for childhood rhabdomyosarcoma. A population-based evaluation.Cancer. 2005 Apr 1; 103(7): 1475-83. http://dx.doi.org/10.1002/cncr.20929

[2] Sultan I, Qaddoumil, Yaser S, et al. Comparing adult and pediatric rhabdomyosarcoma in the surveillance, epidemiology and end results program, 1973 to 2005: an analysis of 2,600 patients. J Clin Oncol. 2009; 27(20): 3391-7. http://dx.doi.org/10.1200/JCO.2008.19.7483

[3] Raney RB, Anderson JR, Barr FG, et al. Rhabdomyosarcoma and undifferentiated sarcoma in the first two decades of life: A selective review of Intergroup rhabdomyosarcoma study group experience and rationale for Intergroup Rhabdomyosarcoma Study V. J Pediatr Hematol Oncol. 2001; 23: 215-220. PMid:11846299. http://dx.doi.org/10.1097/00043426-200105000-00008

[4] Stiller CA, Stevens MCG, Magnani C, et al. Survival of children with soft tissuesarcoma in Europe since 1978: Results from the EUROCARE study. Eur J Cancer. 2001; 37: 767-774. http://dx.doi.org/10.1016/S0959-8049(01)00007-7

[5] Oberlin O, Rey A, Lyden E, et al. Prognostic factors in metastatic rhabdomyosarcomas: results of a pooled analysis. Oncol. 2008; 26: 2384-2389. http://dx.doi.org/10.1200/JCO.2007.14.7207

[6] Newton WA, Gehan EA, Webber BL, et al. Classification of rhabdomyosarcoma and related sarcomas: Pathologic aspects and proposals for a new classification-An Intergroup Rhabdomyosarcoma Study. Cancer. 1995; 76: 1073-1085. http://dx.doi.org/10.1002/1097-0142(19950915)76:6<1073::AID-CNCR2820760624>3.0.CO;2-L

[7] Gerber NK, Wexler LH, Singer S, et al. AdultRhabdomyosarcoma Survival Improved With Treatment on Multimodality Protocols. International Journal of Radiation Oncology Biology Physics. 2013; 86: 58-63. http://dx.doi.org/10.1016/j.ijrobp.2012.12.016

[8] Crist WM, Anderson JR, Meza JL, et al. Intergrouprhabdomyosarcoma study-IV: Results for patients with non metastatic disease. J Clin Oncol. 2001; 19: 3091-4102. PMid:11408506.

[9] Stevens MCG, Rey A, Bouvet N, et al. Treatment of Nonmetastatic Rhabdomyosarcomain Childhood and Adolescence: Third Study of the International Society of Paediatric Oncology-SIOP Malignant Mesenchymal Tumor. JCO. 2005 ; 23 : 12. http://dx.doi.org/10.1200/JCO.2005.08.130

[10] Crist WM, Anderson JR, Meza JL, et al. Intergroup rhabdomyosarcoma study-IV: results for patients with nonmetastatic disease. J Clin Oncol. 2001; 19(12): 3091-102. PMid:11408506

[11] Dantonello TM, Int-Veen C, Harms D, et al. Cooperative trial CWS-91 for localized soft tissue sarcoma in children, adolescents, and young adults. J Clin Oncol. 2009; 27(9): 1446-55. PMid:19224858. http://dx.doi.org/10.1200/JCO.2007.15.0466

[12] Stevens MC, Rey A, Bouvet N, et al. Treatment of nonmetastaticrhabdomyosarcoma in childhood and adolescence: third study of the International Society of Paediatric Oncology - SIOP Malignant Mesenchymal Tumor 89. J Clin Oncol. 2005; 23(12): $2618-28$. http://dx.doi.org/10.1200/JCO.2005.08.130

[13] Ward E, DeSantis C, Robbins A, et al. Childhood and Adolescent Cancer Statistics. CA CANCER J CLIN. 2014 : 1-21. http://dx.doi.org/10.3322/caac.21219

[14] La Quaglia MP, Heller G, Ghavimi F, et al. The effect of age at diagnosis on outcome in rhabdomyosarcoma. Cancer. 1994; 73(1): 109-17. http://dx.doi.org/10.1200/JCO.2005.08.130

[15] Ferrari A, Dileo P, Casanova M, et al. Rhabdomyosarcoma in adults. A retrospective analysis of 171 patients treated at a single institution. Cancer. 2003; 98(3): 571-80. http://dx.doi.org/10.1002/cncr.11550 
[16] Sultan I, Qaddoumi I, Yaser S, et al. Comparing adult and pediatric rhabdomyosarcoma in the surveillance, epidemiology and end results program, 1973 to 2005: an analysis of 2,600 patients. J Clin Oncol. 2009; 27(20): 3391-7.

http://dx.doi.org/10.1200/JCO.2008.19.7483

[17] Van Gaal JC, Van der Graaf WTA, Rikhof B, et al. Age as a prognostic factor for outcome in rhabdomyosarcoma (RMS) patients (pts). In: ASCO annual meeting. 2008.

[18] Joshi D, Anderson JR, Paidas C, et al. Age is an independent prognostic factor in rhabdomyosarcoma: a report from the Soft Tissue Sarcoma Committee of the Children's Oncology Group. Pediatr Blood Cancer. 2004; 42: 64-73. http://dx.doi.org/10.1002/pbc.10441

[19] Arndt CA, Stoner JA, Hawkins DS, et al. Vincristine, actinomycin, and cyclophosphamide compared with vincristine, actionmycin, and cyclophosphamide alternating with vincristine, topotecan, and cyclophosphamide for intermediate-risk rhabdomyosarcoma: Children's oncology group study D9803. J Clin Oncol. 2009; 27: 5182-5188. http://dx.doi.org/10.1200/JCO.2009.22.3768

[20] Walterhouse D, Pappo AS, Meza J, et al. Shorter duration therapy that includes vincristine (V), dactinomycin (A), and lower doses of cyclo- phosphamide (C) with or without radiation therapy for patients withnewly diagnosed low-risk embryonalrh abdomyosarcoma (ERMS): A report from the Children's Oncology Group (COG). J Clin Oncol. 2011; 29 15(S): 9516. http://dx.doi.org/10.1002/cncr.26355

[21] Carola AS Arndt. Risk Stratification of Rhabdomyosarcoma: A Moving Target. ASCO EDUCATIONAL BOOK. $2013: 415-419$. http://dx.doi.org/10.1002/cncr.26355

[22] Hawkins DS, Spunt SL, Skapek SX. Children's Oncology Group's 2013 blueprint for research: Soft tissue sarcomas. Pediatr Blood Cancer. Epub 2012 Dec 19. http://dx.doi.org/10.1002/pbc.24435

[23] Oberlin O, Rey A, Lyden E, et al. Prognostic factors in metastatic rhabdomyosarcomas: results of a pooled analysis. Oncol. 2008; 26: 2384-2389. http://dx.doi.org/10.1200/JCO.2007.14.7207

[24] Maki RG. Pediatric sarcomas occurring in adults. J Surg Oncol. 2008; 97(4): 360-368. http://dx.doi.org/10.1002/jso.20969

[25] Dumont SN, Lazar JA, Bridge RS, et al. PAX3/7-FOXO1 fusion status inolderrhabdomyosarcoma patient population by fluorescent in situ hybridization. J. Cancer Res. Clin. Oncol. 2012; 138: 213-220. http://dx.doi.org/10.1007/s00432-011-1089-7

[26] Sanz N, de Mingo L, Florez F, et al. Rhabdomyosarcoma of the biliary tree. Pediatr Surg Int. 1997; 12: 200-201. http://dx.doi.org/10.1007/BF01350002

[27] Cecchetto G, Carretto E, Bisogno G, et al. Complete second look operation and radiotherapy in locally advanced non-alveolar rhabdomyosarcoma in children: A report from the AIEOP soft tissue sarcoma committee. Pediatr Blood Cancer. 2008; 51(5): 593-597. http://dx.doi.org/10.1002/pbc.21702

[28] Eaton BR, McDonald MW, Kim S, et al. Radiation therapy target volume reduction in pediatric rhabdomyosarcoma: implications for patterns of disease recurrence and overall survival. Cancer. 2013; 119(8): 1578-1585. PMid:23280478. http://dx.doi.org/10.1002/cncr.27934

[29] Wharam MD, Hanfelt JJ, Tefft MC, et al. Radiation therapy for rhabdomyosarcoma: Local failure risk for Clinical Group III patients on Intergroup Rhabdomyosarcoma Study II. Int J Radiat Oncol Biol Phys. 1997; 38: 797-804. http://dx.doi.org/10.1016/S0360-3016(97)00120-X

[30] Gupta AA, Anderson JR, Pappo AS, et al. Patterns of Chemotherapy-Induced Toxicities in Younger Children and Adolescents with Rhabdomyosarcoma. Cancer. 2012 February 15: 1130-37. http://dx.doi.org/10.1002/cncr.26358 